\title{
Application Signal Threshold Adaptation for Vertical Handoff in Heterogeneous Wireless Networks
}

\author{
Ben Liang ${ }^{1}$, Ahmed H. Zahran ${ }^{1}$, and Aladdin O.M. Saleh ${ }^{2}$ \\ 1 Department of Electrical and Computer Engineering, University of Toronto \\ 2 Wireless Technology Planning, Bell Canada
}

\begin{abstract}
In heterogeneous wireless systems, the seamless and efficient handoff between different access technologies (vertical handoff) is essential and remains a challenging problem. The co-existence of access technologies with largely different characteristics results in handoff asymmetry that differs from the traditional intra-network handoff problem. In the case where one network is preferred, the vertical handoff decision should be carefully executed, based on the wireless channel state, network layer characteristics, as well as application requirements. In this paper, we present an adaptive preferred-network lifetime-based handoff strategy, and investigate the effect of an application-based signal strength threshold on the signalling load, available bandwidth, and packet delay. We propose an analytical framework to evaluate the performance of the converged system. We show how the proposed analytical model can be used to provide guidelines for the optimization of vertical handoff in the next generation integrated wireless networks.
\end{abstract}

\section{Introduction}

Wireless technologies are evolving toward broadband information access across multiple networking platforms, in order to provide ubiquitous availability of multimedia applications. Recent trends indicate that wide-area cellular networks based on the 3G standards and wireless Local Area Networks (WLANs) will co-exist to offer multimedia services to end users. By strategically combining these technologies, a converged system can provide both universal coverage and broadband access. Therefore, the integration of heterogeneous networks is expected to become a main focus in the development toward the next generation wireless networks $[1,2,3$.

Mobility management is a main challenge in the converged network [4,5. Both intra-technology handoff and inter-technology handoff take place. Intratechnology handoff is the traditional Horizontal Handoff (HHO) process in which the mobile terminal hands-off between two Access Points (AP) or Base Stations (BS) using the same access technology. On the other hand, inter-technology handoff, or Vertical Handoff (VHO) occurs when the MT roams between different access technologies. The main distinction between VHO and HHO is symmetry. 
While HHO is a symmetric process, VHO is an asymmetric process in which the MT moves between two different networks with different characteristics. This introduces the concept of a preferred network, which is usually the underlay WLAN that provides better throughput performance at lower cost, even if both networks are available and in good condition for the user.

There are two main scenarios in VHO: moving out of the preferred network (MO) and moving into the preferred network (MI) 6]. In either case, it is highly desirable to associate the MT with the preferred network, as long as the preferred network satisfies the user application. This can improve the resource utilization of both access networks, as well as improve the the user perceived QoS. Furthermore, handoff should be seamless with minimum user intervention, while dynamically adapting to the wireless channel state, network layer characteristics, and application requirements.

In this work, we present an adaptive VHO algorithm which takes into consideration the wireless signal strength, handoff latency, and application QoS and delay tolerance. Furthermore, it can satisfy the system handoff signalling load, as well as different application requirements by the tuning of an application specific signal strength parameter. We further propose an analytical model to evaluate the performance of adaptive $\mathrm{VHO}$, which is then applied to show how the VHO decision can be optimized based on multiple conflicting criteria.

\section{Related Work}

The traditional HHO problem has been studied extensively in the past. Several approaches have been considered in cellular networks using the Received Signal Strength (RSS) as an indicator for service availability from a certain point of attachment, as well as for comparison between the current point of attachment and the candidate points of attachment [7]:

- RSS: handoff takes place if the RSS of the candidate point of attachment is larger than the RSS of the current point of attachment $\left(R S S_{n e w}>\right.$ $\left.R S S_{\text {current }}\right)$.

- RSS plus threshold: handoff takes place if the RSS of the candidate point of attachment is larger than the RSS of the current point of attachment and the RSS of the current point of attachment is less than a certain pre-defined threshold $T\left(R S S_{\text {new }}>R S S_{\text {current }}\right.$ and $\left.R S S_{\text {current }}<T\right)$.

- RSS plus hysteresis: handoff takes place if the RSS of the candidate point of attachment is larger than the RSS of the current point of attachment with a pre-defined hysteresis margin $H .\left(R S S_{\text {new }}>R S S_{\text {current }}+H\right)$.

- A dwell timer can be added to any of the above algorithms. In this case, the timer is started when one of the above conditions is satisfied, and the MT performs a handoff if the condition is satisfied for the entire dwell timer interval.

For the VHO process, the RSS's of heterogeneous networks are not comparable due to the asymmetric nature of the handoff problem. However, they can be 
used to determine the availability as well as the condition of different networks. On the one hand, the MI decision should be based on the availability of the WLAN, in satisfactory condition, as well as the user preferences according to predefined policies 8 . On the other hand, the MO decision should maintain efficient utilization of the wireless resources, which implies reliance on the WLAN as far as the network can provide satisfactory service to the user.

As far as we are aware, there are few existing works discussing VHO beyond direct extensions to the common techniques for HHO. Two general directions for VHO algorithms are recorded in the literature. One is based on the traditional strategies of using the RSS that may be combined with other parameters such as network loading. In 9 the authors use the dwell timer as a handoff initiation criterion for their algorithm to extend the residence time of the MT in the WLAN. They combine simulation and analysis to show that associating the MT with the WLAN for the longest possible time result in user throughput improvement even during the transition period in which the RSS oscillates around the receiver sensitivity level. However, they did not define a clear mechanism for choosing the dwell timer value. In [10, Ylianttila et al. present an algorithm that adapts the dwelling timer according to the available data rates in both networks that are defined by the standards. In [11, the same analytical framework of 9 ] is extended to include multiple radio network environments. Their main results show that the effect of the handoff delay seems to be dominant even with the optimal choice of the dwell timer as in [10]. In [12, Zhu and McNair present two cost-based policies for VHO, which considers the available bandwidth and RSS of the available networks. The collective handoff policy estimates one cost for all services, while the prioritized multi-network handoff policy estimates the cost for each service independently.

The second approach uses artificial intelligence techniques [13,14, 15] such as fuzzy logic and neural networks. It is worth mentioning that some of the artificial intelligence based algorithms are complex and may not be easy to implement in practical systems. It is possible to extend our work to include improvement using similar artificial intelligence approaches. However, this is outside the scope of this paper and will be left for future work.

\section{Application Life-Time Adaptation}

\subsection{System Model}

We study the overlapping of $3 \mathrm{G}$ cellular and WLAN networks. The cellular network is assumed to provide universal coverage, while WLAN availability is indicated by the presence of the WLAN beacons 14 that are periodically transmitted by the WLAN AP's. Mobile-IP is assumed for mobility management.

We assume that WLAN hot spots implement loosely coupled connection [16. with the $3 \mathrm{G}$ network using WLAN gateways. These gateways perform several tasks including serving as Mobile-IP agents and possibly providing QoS in the form of multiple service classes defined within the WLAN. However, it is worth mentioning that end-to-end QoS support requires other mechanisms such as 
differentiated services to be implemented over the entire network path. The details of such implementation is unimportant to the proposed VHO algorithm and mathematical analysis. We are mainly concerned about the resultant $\mathrm{VHO}$ delay values.

The MT is equipped with dual interfaces that allow it to communicate with both networks. However, since Mobile-IP provides only one IP tunnel, the MT can connect to one network only at a time. In addition, multi-interface mobility client software is installed on the MT. This software performs Mobile-IP signaling with the foreign and home agents. It periodically scans the available interfaces and measures the observed RSS. Then it intelligently selects the best access network according to the predefined VHO algorithm.

Within the WLAN, a log-linear path loss channel propagation model with shadow fading is used [17]. The RSS is expressed in $\mathrm{dBm}$ as $R S S=P_{T}-L-$ $10 n \log (d)+f(\mu, \sigma)$, where $P_{T}$ is the transmitted power, $L$ is a constant power loss, $n$ is the path loss exponent and usually has values between $2-4, d$ represents the distance of the MT from the $\mathrm{AP}$, and $f(\mu, \sigma)$ represents shadow fading which is modelled as Gaussian with mean $\mu=0$ and standard deviation $\sigma$ with values between 6-12 dB depending on the environment. We assume that when the RSS is below a certain interface sensitivity level, $\alpha$, the MT is unable to communicate with the AP.

\subsection{Adaptive Preferred-Network Life-Time Vertical Handoff}

For the MO scenario when the MT is within a WLAN, we use the RSS to estimate the expected duration after which the MT is unable to maintain its connection with the WLAN. We take into consideration the handoff delay due to MIP tunnelling, authentication, and service initiation. We further consider an Application Signal Strength Threshold (ASST), which is the required level of RSS for the active application to perform satisfactorily.

The ASST is an application dependent parameter which represents a composite of the channel bit error rate, application error resilience, and application QoS requirements. We present here how the ASST can be incorporated into the VHO decision. We further discuss in the next section how the ASST can be adjusted to optimize the overall system performance.

In discrete time, the RSS is expressed as $R S S[k]=\mu_{R S S}[k]+N[k]$, where $k$ is the time index, $\mu_{R S S}[k]=P_{T}-L-10 n \log (d[k])$, and $N[k]=f(\mu, \sigma)$. The averaged RSS, $\overline{R S S}[k]$, can be estimated using a moving average $\overline{R S S}[k]=$ $\frac{1}{W_{\text {average }}} \sum_{i=0}^{W_{\text {average }}{ }^{-1}} R S S[k-i]$. The RSS rate of change, $S[k]$, can be obtained by

$$
S[k]=\frac{M_{1}[k]-M_{2}[k]}{W_{\text {slope }} T_{\text {sampling }}},
$$

where $M_{1}[k]=\frac{2}{W_{\text {slope }}} \sum_{i=0}^{i=\frac{W_{\text {slope }}}{2}-1} \overline{R S S}\left[k-W_{\text {slope }}+1+i\right]$, and $M_{2}[k]=$ $\frac{2}{W_{\text {slope }}} \sum_{i=\frac{W_{\text {slope }}}{2}}^{i=W_{\text {slope }}-1} \overline{R S S}\left[k-W_{\text {slope }}+1+i\right]$. 
Then, we estimate the MT lifetime within the WLAN, $E L[k]$, as follows.

$$
E L[k]=\frac{\overline{R S S}[k]-\gamma}{S[k]},
$$

where $\gamma$ denotes the ASST. Thus, $E L[k]$ represents the application specific time period in which the WLAN is likely to remain usable to the MT.

Based on the measured and estimated parameters, the MT will initiate the MO handoff at time $k$ if the averaged received signal strength is less or equal to a predefined MO threshold, $M O T_{W L A N}$, and the estimated lifetime is less than or equal to the handoff delay threshold, $T_{H O}$. The $M O T_{W L A N}$ is usually chosen to be a few $\mathrm{dB}$ above the wireless interface sensitivity. $T_{H O}$ can be set to the expected handoff delay between the two access technologies. This delay includes several signaling delay components such as discovery delay, authentication delay, and registration delay.

In general, a larger averaging window size results in better estimation but also larger delay in handoff performance [7. Hence, using variable window sizes that adapt to the MT mobility can improve handoff performance. For example, $W_{\text {average }}$ and $W_{\text {slope }}$ can be determined by $W_{\text {average }}=\max \left(10,\left\lfloor\frac{D_{\text {average }}}{V T_{\text {sampling }}}\right\rfloor\right)$ and $W_{\text {slope }}=2 * \max \left(50,\left\lfloor\frac{D_{\text {slope }}}{V T_{\text {sampling }}}\right\rfloor\right)$, where $D_{\text {average }}$ and $D_{\text {slope }}$ represent the averaging and slope distance windows respectively, $T_{\text {sampling }}$ is the sampling interval used in sampling the RSS values, $\lfloor\cdot\rfloor$ represents the greatest lower integer function, and $V$ is the MT velocity away from the AP, which can be obtained by many velocity estimators proposed in the past, for example [18].

In the MI decision, a main factor is the availability of the WLAN, which can be determined by the RSS of the WLAN. Other factors including the QoS, specified in terms of the available bandwidth, security, and user preference can also be considered. In this work, we assume a simplified model where the MT performs MI to the WLAN if $\overline{R S S}[k]>M I T_{W L A N}$ and the available bandwidth is greater than the required bandwidth. In our simulation and analysis, we assume that the WLAN is always in good condition, so that the MT always perform an MI after an unnecessary MO.

In the next section, we provide an analytical framework for evaluating the performance of the proposed cross-layer adaptive vertical handoff.

\section{Performance Analysis}

\subsection{Transition Probabilities}

The calculation of the transition probabilities is based on recursive computation of the handoff probabilities similar to [19]. In the following analysis, we consider

$-P_{W}[k]: \operatorname{Pr}\{\mathrm{MT}$ is associated with the WLAN at instant $\mathrm{k}\}$

$-P_{C}[k]: \operatorname{Pr}\{\mathrm{MT}$ is associated with the $3 \mathrm{G}$ network at instant $\mathrm{k}\}$ 
$-P_{W \mid C}[k]: \operatorname{Pr}\{\mathrm{MT}$ associates itself with the WLAN at instant $\mathrm{k}$ given that it is associated with the cellular network at instant $\mathrm{k}-1\}$

$-P_{C \mid W}[k]: \operatorname{Pr}\{\mathrm{MT}$ associates itself with the $3 \mathrm{G}$ network at instant $\mathrm{k}$ given that it is associated with the WLAN at instant $\mathrm{k}-1\}$

In our model, the MT is assumed to be attached to the WLAN at the beginning; hence $P_{W}[0]=1$ and $P_{C}[0]=0 . P_{W}[k]$ and $P_{C}[k]$ can be calculated recursively:

$$
\begin{aligned}
& P_{W}[k+1]=P_{W \mid C}[k+1] P_{C}[k]+\left(1-P_{C \mid W}[k+1]\right) P_{W}[k], \\
& P_{C}[k+1]=P_{C \mid W}[k+1] P_{W}[k]+\left(1-P_{W \mid C}[k+1]\right) P_{C}[k] .
\end{aligned}
$$

The Conditional probabilities $P_{C \mid W}[k+1]$ and $P_{W \mid C}[k+1]$ depend on the handoff initiation strategy of the algorithm. For the proposed cross-layer adaptive lifetime based VHO algorithm, $P_{C \mid W}[k+1]$ is determined by

$$
P_{C \mid W}[k+1]=\operatorname{Pr}\left\{\overline{R S S}[k+1]<M O T_{W L A N}, E L[k]<T_{H O} \mid W[k]\right\},
$$

where $W[k]$ represents the event that the MT is associated with the WLAN at time $k-1$. In practice, WLAN's are designed for low mobility users. The lifetime part of the MO condition becomes more significant for low mobility users. Hence the MO condition can be reduced to $E L[k]<T_{H O}$. Consequently, one can determine $P_{C \mid W}[k+1]$ as follows:

$$
\begin{aligned}
P_{C \mid W}[k+1] & =\operatorname{Pr}\left\{E L[k+1]<T_{H O} \mid E L[k]>T_{H O}\right\} \\
& =\operatorname{Pr}\left\{\overline{R S S}[k+1]-T_{H O} S[k+1]<\gamma \mid \overline{R S S}[k]-T_{H O} S[k]>\gamma\right\} .
\end{aligned}
$$

Let $Z[k]=\overline{R S S}[k]-T_{H O} * S[k]$. Then we have

$$
P_{C \mid W}[k+1]=\frac{\operatorname{Pr}\{Z[k+1]<\gamma, Z[k]>\gamma\}}{\operatorname{Pr}\{Z[k]>\gamma\}} .
$$

Clearly, since $R S S[k]$ is a Gaussian process, the processes $\overline{R S S}[k]$ and $S[k]$ are Gaussian, and hence $Z[k]$ are Gaussian too. Let its mean be $\mu_{Z}[k]$ and standard deviation be $\sigma_{Z}[k]$. It can be shown that [20]

$$
\mu_{Z}[k]=\mu_{\overline{R S S}}[k]-T_{H O} \mu_{S}[k],
$$

where $\mu_{\overline{R S S}}[k]=\mu_{R S S}[k]+\frac{1}{W_{\text {average }}} \sum_{i=0}^{W_{\text {average }}-1} 10$ nlog $\left(1-\frac{i V T_{\text {sampling }}}{d[k]}\right)$, and $\mu_{S}[k]=\frac{E\left\{M_{1}[k]\right\}-E\left\{M_{2}[k]\right\}}{W_{\text {slope }} T_{\text {sampling }}}$, and furthermore

$$
\sigma_{z}^{2}[k]=\sigma_{\overline{R S S}}^{2}[k]+T_{H O}^{2} \sigma_{S}^{2}[k]+\frac{4 T_{H O} \sigma_{R S S}^{2} \sum_{h=0}^{h=W_{\text {average }}-1}\left(W_{\text {average }}-|h|\right)}{W_{\text {slope }}^{2} T_{\text {sampling }} W_{\text {average }}^{2}}
$$

where $\sigma_{\overline{R S S}}^{2}[k]=\frac{\sigma^{2}}{W_{\text {average }}}$ and $\sigma_{S}^{2}[k]=\frac{4 \sigma^{2}}{\left(T_{\text {sampling }} W_{\text {slope }}^{2} W_{\text {average }}\right)^{2}} \times$ $\left[W_{\text {average }} W_{\text {slope }}+\sum_{h=1}^{W_{\text {average }}-1}\left(W_{\text {average }}-|h|\right)\left(2 W_{\text {slope }}-6|h|\right)\right]$. Additionally, 
$Z[k]$ and $Z[k-1]$ are jointly Gaussian with correlation coefficient $\rho_{Z[k], Z[k-1]}$ as derived in the Appendix, which defines their joint $\operatorname{PDF} f_{Z[k] Z[k-1]}\left(z_{1}, z_{2}\right)$ [21].

Then we can compute $P_{C \mid W}[k+1]$ by

$$
P_{C \mid W}[k+1]=\frac{\int_{-\infty}^{\gamma} \int_{\gamma}^{\infty} f_{Z[k+1] Z[k]}\left(z_{1}, z_{2}\right) d z_{1} d z_{2}}{Q\left(\frac{\gamma-\mu_{Z[k]}[k]}{\sigma_{Z[k]}[k]}\right)}
$$

where $\mathrm{Q}(\mathrm{x})$ is the complementary error function. Similarly, $P_{W \mid C}[k+1]$ can be determined by

$$
P_{W \mid C}[k+1]=\frac{\operatorname{Pr}\{\overline{R S S}[k+1]>M I T, \overline{R S S}[k]<M I T\}}{\operatorname{Pr}\{\overline{R S S}[k]<M I T\}} .
$$

where, similar to the $(\mathrm{Z}[\mathrm{k}+1], \mathrm{Z}[\mathrm{k}])$ tuple, the $(\overline{R S S}[k+1], \overline{R S S}[k])$ tuple is jointly Gaussian. These transition probabilities are used to calculate the performance metrics as follows.

\subsection{Handoff Probabilities and the Number of Handoffs}

The number of handoffs has major impact on the signaling traffic, which may overload the network resulting in degradation in the overall performance. The number of handoffs, denoted $N_{H O}$, is defined as the sum of MO's and MI's between WLAN and $3 \mathrm{G}$ network as the MT roams across the network boundary. Hence, it is a random variable that depends on the instantaneous move out/in probabilities, which can be calculated by

$$
P_{M O}[k+1]=P_{C \mid W}[k+1] P_{W}[k], \quad P_{M I}[k+1]=P_{W \mid C}[k+1] P_{C}[k] .
$$

The MT movement between the two networks can be modeled by a twostate non-homogeneous Markov chain, where each state represents the network with which the MT is associated. The transition probabilities are $P_{M O}[k]$, from WLAN, and $P_{M I}[k]$, from $3 \mathrm{G}$. Hence, by using binary impulse rewards for the handoff transition as shown in [22, we calculate the average accumulated rewards for MO and MI transitions, which are equivalent to the expected number of MO's, $N_{M O}$, and the expected number MI's, $N_{M I}$, respectively. Hence, the expected number of handoffs can be calculated by

$$
E\left\{N_{H O}\right\}=E\left\{N_{M O}\right\}+E\left\{N_{M I}\right\}=\sum_{k=1}^{k_{\max }}\left(P_{M O}[k]+P_{M I}[k]\right)
$$

\subsection{Available Bandwidth}

The available bandwidth to the MT depends on the proportion of time that the MT stays in the WLAN and the 3G network, as well as the state of the WLAN when the MT is connected to the WLAN. To the MT, the WLAN is in one of two states: WLAN Up and WLAN Down. The WLAN Up state represents the event that the WLAN signal received at the MT is above the sensitivity level $\alpha$. 
WLAN Down is the reverse case. Let $p[k]$ be the probability that the WLAN is in the Up state at time $k$. Clearly

$$
p[k]=\operatorname{Pr}\{R S S[k]>\alpha\}=Q\left(\frac{\alpha-\mu[k]}{\sigma}\right) .
$$

Based on the adopted handoff algorithm, the MO distance varies; consequently the captured WLAN Up durations does too. For the rest of the analysis, we are interested in evaluating the system performance during the transition region, which is defined as the range of distance between the point when the RSS starts to oscillate around the interface sensitivity and the WLAN edge. The determination of the transition region is equivalent to a long-standing complex level crossing problem that is analytically tractable only for a few simple cases and is usually solved numerically for complex cases. Here, we obtained the transition region starting bound, denoted $k_{\text {start }}$, from rough estimates based on simulation results.

Then, the WLAN efficiency, $\zeta_{L T}$, defined as the percentage of the WLAN up duration over the MT lifetime in the WLAN, can be estimated as

$$
\zeta_{L T}=\sum_{k=k_{\text {start }}}^{k_{\max }} \overline{P_{M O}}[k] \frac{\sum_{h=1}^{k} p[h]}{k},
$$

where $\overline{P_{M O}}$ is a scaled version of $P_{M O}$ to represent a valid PDF within interval $\left[1, k_{\max }\right]$, and $k_{\max }$ represents the time index at which the MT reaches the WLAN edge and is determined by the planed coverage area.

Hence, the MT available bandwidth, $B W_{A v}$, assuming $R_{W}$ and $R_{C}$ as the effective data rates in WLAN and cellular networks respectively, can be computed as

$$
B W_{A v}=\frac{\zeta_{L T} R_{W}\left(\overline{k_{M O}}-k_{\text {start }}\right)+R_{C}\left(k_{\max }-\overline{k_{M O}}\right)}{\left(k_{\max }-k_{\text {start }}\right)},
$$

where $\overline{k_{M O}}$ denotes the average time to MO.

\subsection{Packet Delay}

In addition to the MT available bandwidth, RSS degradation in the transition region impacts on the head of line (HoL) packet delay probability. To study this, we assume a threshold, $\theta_{D}$ for packet delay in the current hop as a part of the end-to-end delay budget for the real-time application packet from the source to the destination. A packet is considered excessively delayed if its HoL delay exceeds $\theta_{D} 1$. Consequently, the average packet delay probability, $D$, can be estimated as

$$
D=\frac{\sum_{k=k_{\text {start }}}^{\overline{k_{M O}}} P_{D}[k]}{\left(\overline{k_{M O}}-k_{\text {start }}+1\right)}
$$

\footnotetext{
${ }^{1}$ Note that this does not necessarily mean that the packet is lost.
} 
where $P_{D}[k]$ represent the probability that a packet will be excessively delayed, which is equal to the probability of WLAN Down runs whose duration is equal to the delay threshold. Here we have performed an approximation by using $\overline{k_{M O}}$, instead of using $k_{M O}$ and then applying conditional expectation. As shown in the next section, this approximation produces accurate results over a wide range of system parameters.

\section{Numerical Results and Simulation}

\subsection{Simulation Model}

In addition to analysis, we have simulated VHO using MATLAB. The simulation model assumes a MT moving away from the WLAN access point with constant speed $V$. Table 1 shows the values of the simulation parameters. The system parameters are used as in [15. These parameters result in a WLAN coverage of approximately 100 meters in radius. In the following, each point in the simulation results represent the average of 100 runs.

Table 1. Simulation parameters values

\begin{tabular}{|l|c||l|c||l|c||l|c|}
\hline Parameter & Value & Parameter & Value & Parameter & Value & Parameter & Value \\
\hline \hline$P_{T}$ & $100 \mathrm{mWatt}$ & $n$ & 3.3 & $\sigma$ & $7 \mathrm{~dB}$ & $S$ & $28.7 \mathrm{~dB}$ \\
\hline \hline$D_{\text {average }}$ & $0.5 \mathrm{~m}$ & $D_{\text {slope }}$ & $5 \mathrm{~m}$ & $\alpha$ & $-90 \mathrm{dBm}$ & $T_{\text {sampling }}$ & $0.01 \mathrm{sec}$ \\
\hline \hline MOT $_{W L A N}$ & $-85 \mathrm{dBm}$ & $M I T_{W L A N}$ & $-80 \mathrm{dBm}$ & $T_{\text {handoff }}$ & $1 \mathrm{sec}$ & $\frac{R_{W}}{R_{C}}$ & $\frac{25 \mathrm{Mbps}}{2.4 \mathrm{Mbps}}$ \\
\hline
\end{tabular}

\subsection{Performance Comparison}

We compare the performance of adaptive $\mathrm{VHO}$ with traditional hysteresis $\mathrm{VHO}$, which is used in [2]. In hysteresis based algorithms, there are two different thresholds $M I T_{W L A N}$ and $M O T_{W L A N}$ for the MI and MO respectively. The MT performs a MI if the $\overline{R S S}[k]$ is larger than $M I T_{W L A N}$ and performs a MO if $\overline{R S S}[k]$ is smaller than the predefined $M O T_{W L A N}$. Usually, $M I T_{W L A N}$ is chosen larger than $M O T_{W L A N}$ to decrease the number of unnecessary handoffs known as ping-pong effect.

Due to page limitation, we will simply state that the introduction of the adaptive lifetime approach to the traditional hysteresis VHO algorithm results in significant decrease of the number of unnecessary handoffs and significant improvement on the available bandwidth [20]. Clearly, from a pure bandwidth point of view, it is preferable for the MT to perform MO handoff near the WLAN edge, even though the RSS can temporarily go below the MT sensitivity level in the transition region. However, a drawback of increasing the lifetime of the MT within the WLAN is increasing the packet delay resulting from channel degradation. As shown in [20], the packet delay probability using the adaptive approach can be much more than that when the traditional hysteresis algorithm is used. This may be critical if the MT is running real-time application. However, 
by the proper tuning of the ASST as shown in the next subsection, this effect can be adapted to the active real-time application requirements in the MT.

\subsection{Application Signal Strength Threshold Adaptation}

Figures 1, 2, and 3 illustrate the effect of the ASST on the number of handoffs, available bandwidth, and packet delay probability. They show that the number of handoffs decreases when the ASST is reduced, since reducing the ASST allows the MT to remain in the WLAN for a longer duration. For the same reason, the available bandwidth to the MT increases when the ASST is reduced. However, at the same time, the packet delay probability is increased, since signal outage is more severe near the edge of the WLAN. Hence, there is a clear trade off among the handoff signaling load, available bandwidth, and packet delay.

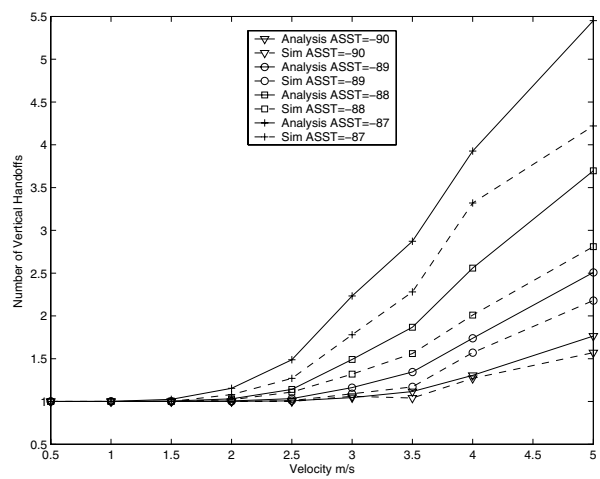

Fig. 1. Number of Handoffs vs. ASST

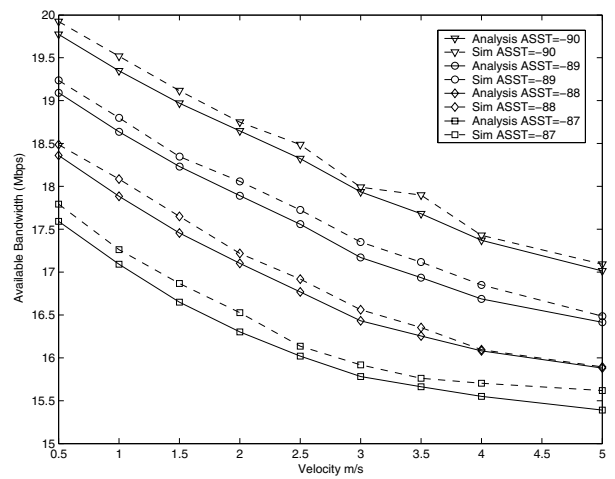

Fig. 2. Available Bandwidth vs. ASST

Clearly, the ASST should not depend on the application QoS alone. Rather, it can be optimally tuned based on the various conflicting criteria of VHO. Likewise, the optimal VHO decision can be made adaptive to the RSS variation, network delay characteristics, and application QoS demands, through a properly chosen ASST value. The propose analytical framework provides a means to carry out this optimization.

As an example, a possible cost function to aggregate the multiple VHO criteria may be $C_{\text {total }}=\frac{c_{H} E\left\{N_{O H}\right\}+c_{D} D}{B W_{a v}}$, where $c_{H}$ represents the signaling cost per handoff, $c_{D}$ represents the penalty factor for packet delay, and $C_{\text {total }}$ is normalized to cost per Mbps of data bandwidth 2 .

Figure 4 plots $C_{\text {total }}$ over different ASST values, for $V=2, c_{H}=100$, and $c_{D}=10000$, where each curve represents a delay threshold value of $40 \mathrm{~ms}$,

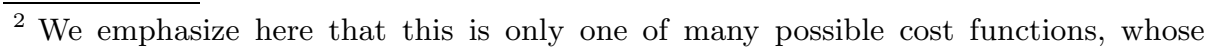
suitability depends on practical application goals and system constraints. 


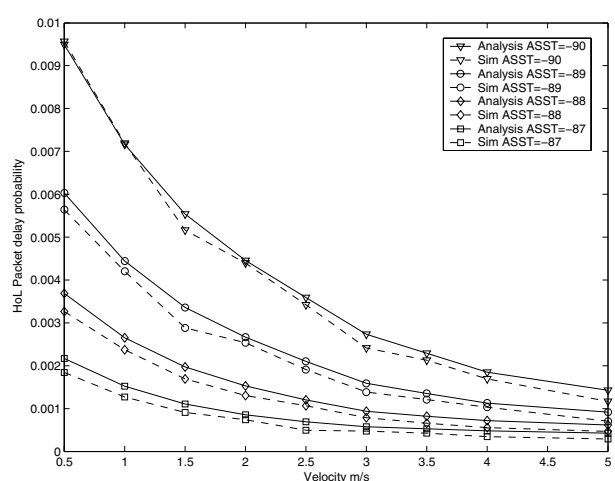

Fig. 3. HoL Packet Delay Rate vs. ASST $\left(\theta_{D}=30 \mathrm{~ms}\right)$

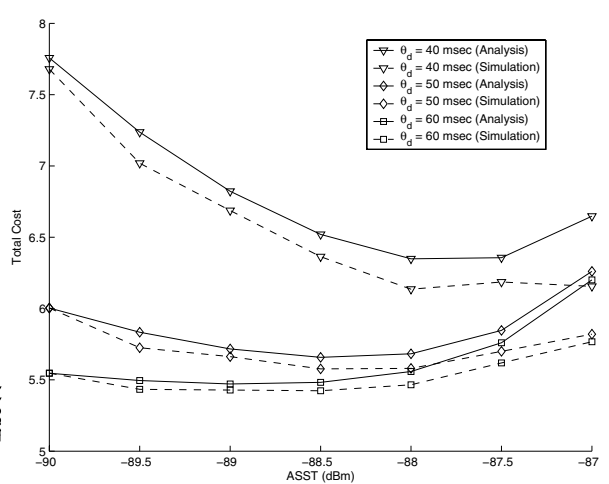

Fig. 4. Total Cost vs. ASST $(V=2)$

$50 \mathrm{~ms}$, and $60 \mathrm{~ms}$, respectively. Clearly, the optimal ASST increases as the delay threshold decreases. In particular, when $\theta_{D}=40 \mathrm{~ms}$, an optimal ASST of $-88 \mathrm{dBm}$ strikes the optimal balance to minimize the total cost, but when $\theta_{D}=60 \mathrm{~ms}$, the optimal ASST is $-89 \mathrm{dBm}$. To implement this in practice, a lookup table can be built based on the proposed numerical analysis results.

\section{Conclusions}

We have presented a cross-layer adaptive VHO approach that takes into account the wireless channel variation, network layer latency, and application QoS demands. We have proposed an analytical framework to evaluate the performance of VHO based on multiple criteria. The proposed application signal threshold adaptation provides a means for flexible system design. Given a predefined priority policy, it can be used to optimize the tradeoff between handoff signalling, available bandwidth, and packet delay. Since the ASST can be optimally tuned for any access network based on practical system characteristics and requirements, it may have a significant role in future generation wireless networks where access technologies with vastly differing characteristics are expected to seamlessly co-exist and efficiently inter-operate.

\section{References}

1. Berezdivin, R., Breinig, R., Topp, R.: Next-generation wireless communications concepts and technologies. IEEE Communications Magazine (2002)

2. Buddhikot, M.M., Chandranmenon, G., Han, S., Lee, Y.W., Miller, S., Salgarelli, L.: Integration of 802.11 and third generation wireless data networks. In: Proc. of IEEE INFOCOM, San Francisco, US (2003) 503-512 
3. Salkintzis, A.K., Fors, C., Pazhyannur, R.: WLAN-GPRS integration for nextgeneration mobile data networks. IEEE Wireless Commun. 9 (2002) 112-124

4. Akyildiz, I.F., McNair, J., Ho, J., Uzunalioglu, H., Wang, W.: Mobility management in current and future communications networks. IEEE Network 12 (1998) $39-49$

5. Liang, B., Haas, Z.J.: Predictive distance-based mobility management for multidimensional pcs networks. IEEE/ACM Transactions on Networking 11 (2003) 718-732

6. Makela, J., Ylianttila, M., Pahlavan, K.: Handoff decision in multi-service networks. In: Proc. of 11th IEEE International Symposium on Personal, Indoor and Mobile Radio Communications (PIMRC'00). Volume 1., London, UK (2000) 655-659

7. Pollini, G.P.: Trends in handover design. IEEE Commun. Mag. 34 (1996) 82-90

8. Wang, H., Katz, R.H., Giese, J.: Policy-enabled handoffs across heterogeneous wireless networks. In: Proc. of the Second IEEE Workshop on Mobile Computer Systems and Applications, New Orleans, Louisiana (1999) 51

9. Hatami, A., Krishnamurthy, P., Pahlavan, K., Ylianttila, M., Makela, J., Pichna, R.: Analytical framework for handoff in non-homogeneous mobile data networks. In: Proc. of (PIMRC'99), Osaka, Japan, (1999) 760-764

10. Ylianttila, M., Pande, M., Makela, J., Mahonen, P.: Optimization scheme for mobile users performing vertical hand-offs between IEEE 802.11 and GPRS/EDGE networks. In: Proc. of IEEE Global Telecommunications Conference GLOECOM'01. Volume 6., San Antonio, Texas, USA (2001) 3439-3443

11. Ylianttila, M., Makela, J., Mahonen, P.: Supporting resource allocation with vertical handoffs in multiple radio network environment. In: Proc. of IEEE International Symposium on Personal, Indoor and Mobile Radio Com-munications (PIMRC'02), Lisbon, Portugal (2002) 64-68

12. Zhu, F., McNair, J.: Optimizations for vertical handoff decision algorithms. In: in Proc. of IEEE Wireless Communications and Networking Conference (WCNC). Volume 2. (2004) 867-872

13. Ylianttila, M., Pichna, R., Vallstram, J., Makela, J., Zahedi, A., Krishnamurthy, P., Pahlavan, K.: Handoff procedure for heterogeneous wireless networks. In: Proc. of IEEE Global Telecommunications Conference (GLOBECOM'99). Volume 5. (1999) 2783-2787

14. Pahlavan, K., Krishnamurthy, P., Hatami, A., Ylianttila, M., Makela, J.P., Pichna, R., Vallstron, J.: Handoff in hybrid mobile data networks. IEEE Commun. Mag. 7 (2000) 34-47

15. Majlesi, A., Khalaj, B.H.: An adaptive fuzzy logic based handoff algorithm for hybrid networks. In: Proc. of 6th International Conference on Signal Processing. Volume 2. (2002) 1223-1228

16. Buddhikot, M.M., Chandranmenon, G., Han, S., Lee, Y., Miller, S., Salgarelli, L.: Design and implementation of a WLAN/CDMA2000 interworking architecture. IEEE Commun. Mag. 41 (2003) 90-100

17. Rappaport, T.S.: Wireless Comm.: Principles and Practice. Prentice Hall (1999)

18. Tepedelenlioglu, C., Giannakis, G.: On velocity estimation and correlation properties of narrow-band mobile communication channels. IEEE Trans. on Vehicular Technology 50 (2001) 1039- 1052

19. Zhang, N., Holtzman, J.M.: Analysis of handoff algorithms using both absolute and relative measurements. IEEE Transactions on Vehicular Technology 45 (1996) $174-179$ 
20. Zahran, A.H., Liang, B.: ALIVE-HO: Adaptive lifetime vertical handoff for heterogeneous wireless networks. Technical report, University of Toronto (2004)

21. Papoulis, A., Pillai, S.: Probability, Random Variables and Stochastic Processes. 4th edn. McGraw-Hill (2002)

22. Bolch, G., Greiner, S., de Meer, H., Trivedi, K.S.: Queuing networks and Markov Chains: Modeling and Performance Evaluation with Computer Science Applications. 2nd edn. Wiley (1998) 